Groups Geom. Dyn. 9 (2015), 811-830

DOI $10.4171 / \mathrm{GGD} / 329$
Groups, Geometry, and Dynamics

(C) European Mathematical Society

\title{
Weak density of orbit equivalence classes of free group actions
}

\author{
Lewis Bowen
}

\begin{abstract}
It is proven that the orbit-equivalence class of any essentially free probabilitymeasure-preserving action of a free group $G$ is weakly dense in the space of actions of $G$.
\end{abstract}

Mathematics Subject Classification (2010). 37A20, 37A15.

Keywords. Orbit equivalence, free groups, weak equivalence.

\section{Introduction}

The results of this paper are motivated by orbit-equivalence theory and weak equivalence of group actions. Let us first recall some terminology before delving into background material.

Let $(X, \mu)$ be a standard non-atomic probability space and $\operatorname{Aut}(X, \mu)$ the group of all measure-preserving automorphisms of $(X, \mu)$ in which we identify two automorphisms if they agree on a conull subset. Let $G$ be a countable group. By an action of $G$ we will mean a homomorphism $a: G \rightarrow \operatorname{Aut}(X, \mu)$. In particular, all actions in this paper are probability-measure-preserving. Let $A(G, X, \mu)$ denote the set of all such actions. It admits a natural topology as follows. First, let us recall that $\operatorname{Aut}(X, \mu)$ is a Polish group with the weak topology (see $\S 2$ for details). We endow the product space $\operatorname{Aut}(X, \mu)^{G}$ with the product topology and view $A(G, X, \mu)$ as a subspace $A(G, X, \mu) \subset \operatorname{Aut}(X, \mu)^{G}$ with the induced topology. It is well-known that $A(G, X, \mu)$ is a Polish space [18].

1.0.1. Weak containment. If $a \in A(G, X, \mu)$ and $T \in \operatorname{Aut}(X, \mu)$, define $a^{T} \in$ $A(G, X, \mu)$ by

$$
a^{T}(g)=T a(g) T^{-1} .
$$

Let

$$
[a]_{M C}=\left\{a^{T}: T \in \operatorname{Aut}(X, \mu)\right\} \subset A(G, X, \mu)
$$

be the measure-conjugacy class of a. 
Given two actions $a, b \in A(G, X, \mu)$ we say $a$ is weakly contained in $b$ (denoted $a \prec b$ ) if $a$ is contained in the closure of the measure-conjugacy class of $b$ (i.e., $a \in \overline{[b]_{M C}}$ ). We say $a$ is weakly equivalent to $b$ if $a \prec b$ and $b \prec a$. These notions were introduced by A. Kechris [18] as an analog of weak containment of unitary representations.

We can also think of weak equivalence as describing the manner in which the Rohlin Lemma fails for non-amenable groups. Recall that the Rohlin Lemma states that any pmp $\mathbb{Z}$-action is approximately periodic. This fundamental fact is critically important in much classical ergodic theory. It has been extended to amenable groups [24]. Moreover, the Rohlin Lemma is essentially equivalent to the statement that if $G$ is infinite and amenable then any essentially free action $a \in A(G, X, \mu)$ weakly contains all actions of $G$ (i.e., $[a]_{M C}$ is dense in $A(G, X, \mu))$ [19]. By contrast, any non-amenable group admits an essentially free strongly ergodic action (e.g., Bernoulli shift actions) [27, 21]. By definition, the closure of the measure-conjugacy class of a strongly ergodic action cannot contain any non-ergodic action. So each non-amenable group admits at least two non-weakly-equivalent essentially free actions. It is an open problem whether any non-amenable group admits at least two ergodic non-weakly-equivalent actions. However M. Abert and G. Elek [1] made use of profinite actions to show that there is an explicit large family of residually finite groups $G$ that admit an uncountable family of ergodic non-weakly-equivalent actions. This family includes non-abelian free groups.

1.0.2. Orbit-equivalence. We say two actions $a, b \in A(G, X, \mu)$ are orbit-equivalent if there exists $T \in \operatorname{Aut}(X, \mu)$ which takes orbits to orbits: $T(a(G) x)=$ $b(G) T(x)$ for a.e. $x \in X$. We say that $a \in A(G, X, \mu)$ is essentially free if for a.e. $x \in X$ the stabilizer of $x$ in $G$ is trivial: $\{g \in G: a(g) x=x\}=\left\{e_{G}\right\}$.

If $G$ is amenable then every two essentially free ergodic actions of $G$ are orbit equivalent [24]. On the other hand, I. Epstein proved that if $G$ is non-amenable then $G$ admits an uncountable family of essentially free non-orbit-equivalent ergodic pmp actions $[10,16]$. This followed a series of earlier results that dealt with various important classes of non-amenable groups [13, 15, 17, 22, 23, 25]. In [16] it shown that essentially free mixing actions of any non-amenable group $G$ cannot be classified by orbit-equivalence up to countable structures.

The main result of this paper shows that, although there are uncountably many essentially free non-orbit-equivalent ergodic pmp actions of any non-abelian free group, the orbit-equivalence class of any such action is dense in the space of all actions. 
1.0.3. Results. Our main result is:

Theorem 1.1. Let $G$ be a free group with at most countably many generators. Let $A_{\text {free }}(G, X, \mu) \subset A(G, X, \mu)$ denote the subset of all essentially free actions. For any $a \in A(G, X, \mu)$, let $[a]_{O E}$ denote the set of all actions $b \in A(G, X, \mu)$ which are orbit-equivalent to $a$. Then for any $a \in A_{\text {free }}(G, X, \mu),[a]_{O E} \cap A_{\text {free }}(G, X, \mu)$ is dense in $A(G, X, \mu)$.

By contrast we can use deep rigidity results due to $\mathrm{S}$. Popa [25, 26] and Y. Kida [22] to show that many groups do not satisfy Theorem 1.1. For this purpose, let us recall that if $(K, \kappa)$ is a probability space then any countable group $G$ acts on the product space $(K, \kappa)^{G}$ by

$$
(g x)(f)=x\left(g^{-1} f\right), \quad x \in K^{G}, g, f \in G .
$$

This action is called the Bernoulli shift over $G$ with base space $(K, \kappa)$.

Theorem 1.2. Let $G$ be any countably infinite group satisfying at least one of the following conditions:

(1) $G=G_{1} \times G_{2}$ where $G_{1}, G_{2}$ are both infinite, $G_{1}$ is nonamenable and $G$ has no nontrivial finite normal subgroups;

(2) $G$ is the mapping class group of the genus $g$-holed surface for some $(g, n)$ with $3 g+n-4>0$ and $(g, n) \notin\{(1,2),(2,0)\}$;

(3) $G$ has property $(T)$ and every nontrivial conjugacy class of $G$ is infinite.

Let $(X, \mu)$ be a standard non-atomic probability space and let $a \in A(\Gamma, X, \mu)$ be isomorphic to the Bernoulli action $G \curvearrowright([0,1], \lambda)^{G}$ where $\lambda$ is the Lebesgue measure on the unit interval $[0,1]$. Then $[a]_{O E} \cap A_{\text {free }}(G, X, \mu)$ is not dense in $A(G, X, \mu)$.

Before proving this, we need a lemma.

Definition 1. Let $a \in A(G, X, \mu)$ and $\alpha \in \operatorname{Aut}(G)$. Observe that the composition $a \circ \alpha \in A(G, X, \mu)$. We say that two actions $a, b \in A(G, X, \mu)$ are conjugate up to automorphisms if there exists $\alpha \in \operatorname{Aut}(G)$ and $T \in \operatorname{Aut}(X, \mu)$ such that $b=(a \circ \alpha)^{T}$.

Lemma 1.3. Let $G$ be any countable group, $(K, \kappa)$ a standard probability space and $G \curvearrowright^{a}(K, \kappa)^{G}$ the Bernoulli shift action. $\left(S o(g x)(f)=x\left(g^{-1} f\right)\right.$ for $x \in K^{G}$ and $g, f \in G)$. Then any action of $G$ which is conjugate to a up to automorphisms is measurably conjugate to a. 
Proof. Suppose $\alpha \in \operatorname{Aut}(G)$. It suffices to show that $a$ is measurably conjugate to $a \circ \alpha$. For this purpose, define

$$
T: K^{G} \longrightarrow K^{G}
$$

by

$$
T(x)(g)=x\left(\alpha^{-1}(g)\right) .
$$

Then for any $g, f \in G$ and $x \in K^{G}$,

$$
\begin{aligned}
T(f x)(g) & =(f x)\left(\alpha^{-1}(g)\right) \\
& =x\left(f^{-1} \alpha^{-1}(g)\right) \\
& =x\left(\alpha^{-1}\left(\alpha\left(f^{-1}\right) g\right)\right) \\
& =(T x)\left(\alpha\left(f^{-1}\right) g\right) \\
& =\alpha(f)(T x)(g) .
\end{aligned}
$$

This shows that $T$ intertwines $a$ and $a \circ \alpha$ as required.

Proof of Theorem 1.2. Using the previous lemma and [26, Corollary 1.3], [22, Theorem 1.4] and [25, Corollary 0.2] we obtain that if $G$ and $a$ are as above then $[a]_{O E} \cap A_{\text {free }}(G, X, \mu)=[a]_{M C}$. Moreover $a$ is strongly ergodic [21]. So there does not exist any non-ergodic actions in the closure of its measure-conjugacy class. In particular $[a]_{M C}$ is not dense in $A(G, X, \mu)$.

Remark 1. Theorem 1.1 and the upper semi-continuity of cost [18, Theorem 10.12] imply that finitely generated free groups have fixed price, a fact originally obtained by Gaboriau [12].

Remark 2. Because free groups are residually finite and therefore sofic, Theorem 1.1 implies that the orbit-equivalence relation of every essentially free $a \in$ $A(\Gamma, X, \mu)$ is sofic. This fact was first obtained in [9] (it can also be obtained as a consequence of property MD for free groups [19] which was discovered earlier in a different context in [4]). A new proof of this appears in [3].

Question 1. Which groups $\Gamma$ satisfy the conclusion of Theorem 1.1? For example, do all strongly treeable groups satisfy this conclusion? Does $P S L(2, \mathbb{R})$ satisfy the conclusion? 
Question 2. Are orbit-equivalence classes meager? That is, is the set $[a]_{\text {OE }}$ from Theorem 1.1 meager in $A(G, X, \mu)$ ? If so, then combined with ideas and results of [16] it should be possible to prove that if $G$ is a nonabelian free group then for any comeager subset $Y \subset A(G, X, \mu)$ it is not possible to classify actions in $Y$ by orbit-equivalence up to countable structures.

1.1. A special case. To give the reader a feeling for the proof of Theorem 1.1 , we show how to quickly prove a special case.

Theorem 1.4. Let $G$ be a finitely generated free group and $a \in A(G, X, \mu)$ be essentially free. Let $S \subset G$ be a free generating set. Suppose that for every $s \in S$, the automorphism $a(s) \in \operatorname{Aut}(X, \mu)$ is ergodic. Then $[a]_{O E} \cap A_{\text {free }}(G, X, \mu)$ is dense in $A(G, X, \mu)$.

We need two lemmas first.

Lemma 1.5. Suppose that $T \in \operatorname{Aut}(X, \mu)$ is ergodic, $\epsilon>0$ and $\left\{C_{i}\right\}_{i<k},\left\{D_{i}\right\}_{i<k}$ are two measurable partitions of $X$ such that for each $i<k, C_{i}$ and $D_{i}$ have the same measure. Then there is a $T^{\prime} \in \operatorname{Aut}(X, \mu)$ with the same orbits as $T$ such that for all $i$ the measure of $T^{\prime}\left(C_{i}\right) \triangle D_{i}$ is less than $\epsilon$.

Proof. This is [11, Lemma 8].

Lemma 1.6. Let $H_{1}, \ldots, H_{n}$ be countable groups and $G=H_{1} * \cdots * H_{n}$ be their free product. Let $a \in A_{\text {free }}(G, X, \mu), a^{\prime} \in A(G, X, \mu)$ and suppose that for every $i,\left(a^{\prime} \uparrow H_{i}\right)$ is essentially free and $\left(a \uparrow H_{i}\right)$ and $\left(a^{\prime} \uparrow H_{i}\right)$ have essentially the same orbits. Then $a^{\prime}$ is essentially free.

Proof. If $g \in G$ is nontrivial then $g=t_{1} \cdots t_{n}$ for some $t_{i} \in H_{j_{i}} \backslash\{e\}$ with $j_{i} \neq j_{i+1}$ for $i<n$. For a.e. $x \in X, a_{g}^{\prime} x=a_{h} x$ for some $h \in G$ of the form $h=s_{1} \cdots s_{n}$ with $s_{i} \in H_{j_{i}}$. Since $\left(a^{\prime} \uparrow H_{i}\right)$ is essentially free, $s_{i}$ is nontrivial for each $i$. Since $a$ is essentially free, $a_{h} x \neq x$ almost surely, so $a_{g}^{\prime} x \neq x$ almost surely. Since $g$ is arbitrary, $a^{\prime}$ is essentially free.

Proof of Theorem 1.4. Let $b \in A(G, X, \mu)$. We will show that $b \in \overline{[a]_{O E}}$. By Lemma 2.3 below, it suffices to show that if $\left\{C_{i}\right\}_{i<k}$ is a measurable partition of $X$ and $\epsilon>0$ then there exists a measurable partition $\left\{D_{i}\right\}_{i<k}$ of $X$ and an action $a^{\prime} \in[a] O E$ such that

$$
\left|\mu\left(C_{i} \cap b_{s} C_{j}\right)-\mu\left(D_{i} \cap a_{s}^{\prime} D_{j}\right)\right|<\epsilon
$$

for every $s \in S$ and $1 \leq i, j<k$ (where for example $b_{s}=b(s)$ ). 
By Lemma 1.5 for every $s \in S$ there is an automorphism $a_{s}^{\prime} \in \operatorname{Aut}(X, \mu)$ with the same orbits as $a_{s}$ such that

$$
\mu\left(a_{s}^{\prime}\left(C_{i}\right) \Delta b_{s}\left(C_{i}\right)\right)<\epsilon .
$$

Therefore, equation (1) holds with $D_{i}=C_{i}$ for all $i$. It is easy to verify that $a^{\prime}$ is orbit-equivalent to $a$ (indeed $a^{\prime}$ has the same orbits as $a$ ). By Lemma 1.6, $a^{\prime} \in A_{\text {free }}(G, X, \mu)$.

The conclusion of Lemma 1.5 does not hold in general if $T$ is non-ergodic. In order to prove Theorem 1.1 we will show instead that if the sets $\left\{C_{i}\right\}_{i<k}$ are sufficiently equidistributed with respect to the ergodic decomposition of $T$ then we can find an automorphism $T^{\prime}$ with the same orbits as $T$ such that the numbers $\mu\left(C_{i} \cap T^{\prime} C_{j}\right)$ are close to any pre-specified set of numbers satisfying the obvious restrictions.

Acknowledgements. Thanks to Robin Tucker-Drob for pointing me to the reference [11, Lemma 8]. I am partially supported by NSF grant DMS-0968762 and NSF CAREER Award DMS-0954606.

\section{The weak topology}

Here we review the weak topology and obtain some general results regarding weak containment. So let $(X, \mu)$ be a standard non-atomic probability space. The measure algebra of $\mu$, denoted MALG $(\mu)$, is the collection of all measurable subsets of $X$ modulo sets of measure zero. There is a natural distance function on the measure-algebra defined by

$$
d(A, B)=\mu(A \triangle B)
$$

for any $A, B \in \operatorname{MALG}(\mu)$. Because $\mu$ is standard, there exists a dense sequence $\left\{A_{i}\right\}_{i=1}^{\infty} \subset \operatorname{MALG}(\mu)$. Using this sequence we define the weak-distance between elements $T, S \in \operatorname{Aut}(X, \mu)$ by

$$
d_{w}(T, S)=\sum_{i=1}^{\infty} 2^{-i} \mu\left(T A_{i} \Delta S A_{i}\right) .
$$

The topology induced by this distance is called the weak topology. While $d_{w}$ depends on the choice of $\left\{A_{i}\right\}_{i=1}^{\infty}$, the topology on $\operatorname{Aut}(X, \mu)$ does not depend on this choice. 
Let $G$ be a countable group. Recall that $A(G, X, \mu)$ denotes the set of all homomorphisms $a: G \rightarrow \operatorname{Aut}(X, \mu)$. We may view $A(G, X, \mu)$ as a subset of the product space $\operatorname{Aut}(X, \mu)^{G}$ from which it inherits a Polish topology [18].

Notation 1. If $v \in A(G, X, \mu)$ and $g \in G$ then we write $v_{g}=v(g)$.

Lemma 2.1. Let $G$ be a countable group. Let $v \in A(G, X, \mu)$ and $W \subset A(G, X, \mu)$. Then $v$ is in the closure $\bar{W}$ if and only if, for every $\epsilon>0$, for every finite Borel partition $\mathcal{P}=\left\{P_{1}, \ldots, P_{n}\right\}$ of $X$ and every finite set $F \subset G$ there exists $w \in W$ and a finite Borel partition $Q=\left\{Q_{1}, \ldots, Q_{n}\right\}$ of $X$ such that

$$
\left|\mu\left(P_{i} \cap v_{g} P_{j}\right)-\mu\left(Q_{i} \cap w_{g} Q_{j}\right)\right|<\epsilon
$$

for every $g \in F$ and $1 \leq i, j \leq n$.

Proof. This is essentially the same as [18, Proposition 10.1]. It also follows from [5, Theorem 1].

Corollary 2.2. In order to prove Theorem 1.1, it suffices to prove the special case in which $G$ is finitely generated.

Proof. Let $G$ be a countably generated free group with free generating set $S=\left\{s_{1}, s_{2}, \ldots\right\} \subset G$. Let $a, b \in A(G, X, \mu)$ and suppose $a$ is essentially free. Let $\epsilon>0, \mathcal{P}=\left\{P_{1}, \ldots, P_{n}\right\}$ be a Borel partition of $X$ and $F \subset G$ be finite. By Lemma 2.1 it suffices to show there exists $a^{\prime} \in[a]_{O E} \cap A_{\text {free }}(G, X, \mu)$ and a finite Borel partition $Q=\left\{Q_{1}, \ldots, Q_{n}\right\}$ of $X$ such that

$$
\left|\mu\left(P_{i} \cap b_{g} P_{j}\right)-\mu\left(Q_{i} \cap a_{g}^{\prime} Q_{j}\right)\right|<\epsilon
$$

for every $g \in F$ and $1 \leq i, j \leq n$. Let $G_{n}<G$ be the subgroup generated by $\left\{s_{1}, \ldots, s_{n}\right\}$. Choose $n$ large enough so that $F \subset G_{n}$. Because we are assuming Theorem 1.1 is true for finitely generated free groups, there exists an action $a^{\prime \prime} \in$ $A_{\text {free }}\left(G_{n}, X, \mu\right)$ orbit-equivalent to $\left.a\right|_{G_{n}}$ such that

$$
\left|\mu\left(P_{i} \cap b_{g} P_{j}\right)-\mu\left(Q_{i} \cap a_{g}^{\prime \prime} Q_{j}\right)\right|<\epsilon
$$

for every $g \in F$ and $1 \leq i, j \leq n$. By definition of orbit-equivalence, there exists an automorphism $T \in \operatorname{Aut}(X, \mu)$ such that $a^{\prime \prime}$ and $\left(\left.a\right|_{G_{n}}\right)^{T}$ have the same orbits. 
Define $a^{\prime} \in A(G, X, \mu)$ by

$$
a^{\prime}\left(s_{i}\right)= \begin{cases}a^{\prime \prime}\left(s_{i}\right) & \text { if } 1 \leq i \leq n, \\ \operatorname{Ta}\left(s_{i}\right) T^{-1} & \text { for } i>n .\end{cases}
$$

Then clearly $a^{\prime}$ is orbit-equivalent to $a$ and $a^{\prime}$ satisfies (2) because of (3). By Lemma 1.6, $a^{\prime}$ is essentially free.

The next result implies that we can replace the finite set $F \subset G$ appearing in the lemma above with a fixed generating set $S \subset G$. This is crucial to the whole approach because it allows us to reduce Theorem 1.1 from a problem about actions of the free group to a problem about actions of the integers.

Lemma 2.3. Let $G$ be a group with a finite symmetric generating set $S$. Let $v \in$ $A(G, X, \mu)$ and $W \subset A(G, X, \mu)$. Suppose that for every $\epsilon>0$ for every finite Borel partition $\mathcal{P}=\left\{P_{1}, \ldots, P_{n}\right\}$ of $X$ there exists $w \in W$ and a finite Borel partition $Q=\left\{Q_{1}, \ldots, Q_{n}\right\}$ of $X$ such that

$$
\left|\mu\left(P_{i} \cap v_{s} P_{j}\right)-\mu\left(Q_{i} \cap w_{s} Q_{j}\right)\right|<\epsilon
$$

for every $s \in S$ and $1 \leq i, j \leq n$. Then $v \in \bar{W}$.

Proof. Let $\epsilon>0, \mathcal{P}=\left\{P_{1}, \ldots, P_{n}\right\}$ be a Borel partition of $X$ and $F \subset G$ be a finite set. By Lemma 2.1 it suffices to show that there exists $w \in W$ and a finite Borel partition $\mathcal{Q}=\left\{Q_{1}, \ldots, Q_{n}\right\}$ of $X$ such that

$$
\left|\mu\left(P_{i} \cap v_{g} P_{j}\right)-\mu\left(Q_{i} \cap w_{g} Q_{j}\right)\right|<\epsilon
$$

for every $g \in F$ and $1 \leq i, j \leq n$.

In order to do this, we may assume that for some integer $r \geq 0, F=B(e, r)$ is the ball of radius $r$ centered at the identity in $G$ with respect to the word metric $d_{S}(\cdot, \cdot)$ induced by the generating set $S$.

Let $\mathcal{P}^{\prime}=\bigvee_{g \in F} g \mathcal{P}$ be the common refinement of the partitions $\{g \mathcal{P}: g \in F\}$. By hypothesis, there exists a partition $\mathcal{Q}^{\prime}$ of $X$, a bijection $\beta: \mathcal{P}^{\prime} \rightarrow Q^{\prime}$ and an action $w \in W$ such that

$$
\left|\mu\left(P^{\prime} \cap v_{s} P^{\prime \prime}\right)-\mu\left(\beta\left(P^{\prime}\right) \cap w_{s} \beta\left(P^{\prime \prime}\right)\right)\right|<\epsilon\left|\mathcal{P}^{\prime}\right|^{-2}|F|^{-1} / 4
$$

for every $P^{\prime}, P^{\prime \prime} \in \mathcal{P}^{\prime}$ and $s \in S$. 
Let $\Sigma\left(\mathcal{P}^{\prime}\right)$ denote the sigma-algebra generated by $\mathcal{P}^{\prime}$ (and define $\Sigma\left(Q^{\prime}\right)$ similarly). There is a unique boolean-algebra homomorphism from $\Sigma\left(\mathcal{P}^{\prime}\right)$ to $\Sigma\left(\mathrm{Q}^{\prime}\right)$ extending $\beta$. We also let $\beta$ denote this homomorphism.

Let $\mathcal{Q}=\{\beta(P): P \in \mathcal{P}\}$. It is immediate that $Q$ is a finite Borel partition of $X$. We will show that it satisfies (4).

Claim 1. $|\mu(\beta(P))-\mu(P)|<\epsilon / 2$ for every $P \in \Sigma\left(\mathcal{P}^{\prime}\right)$.

Proof of Claim 1. Let $s \in S$. By (5)

$$
|\mu(\beta(P))-\mu(P)| \leq \sum_{P^{\prime}, P^{\prime \prime}}\left|\mu\left(P^{\prime} \cap v_{s} P^{\prime \prime}\right)-\mu\left(\beta\left(P^{\prime}\right) \cap w_{s} \beta\left(P^{\prime \prime}\right)\right)\right|<\epsilon / 2
$$

where the sum is over all $P^{\prime}, P^{\prime \prime} \in \mathcal{P}^{\prime}$ with $P^{\prime} \subset P$.

Claim 2. $\mu\left(\beta\left(v_{g} P\right) \Delta w_{g} \beta(P)\right) \leq \epsilon|g| /(2|F|)$ for all $P \in \mathcal{P}$ and $g \in F$ where $|g|$ denotes the word length of $g$. Moreover equality holds only in the case $|g|=0$.

Proof of Claim 2. We prove this by induction on the word length $|g|$. It is obviously true when $|g|=0$. So we assume there is an integer $m \geq 0$ such that the statement is true for all $g$ with $|g| \leq m$. Now suppose that $|g|=m+1$ and $g \in F$. Then $g=s h$ for some $h \in F$ and $s \in S$ such that $|h|=m$. By induction,

$$
\begin{aligned}
\mu & \left(\beta\left(v_{g} P\right) \Delta w_{g} \beta(P)\right) \\
& =\mu\left(\beta\left(v_{s h} P\right) \Delta w_{s h} \beta(P)\right) \\
& =\mu\left(\beta\left(v_{s h} P\right) \Delta w_{s} \beta\left(v_{h} P\right)\right)+\mu\left(w_{s} \beta\left(v_{h} P\right) \Delta w_{s h} \beta(P)\right) \\
& =\mu\left(\beta\left(v_{s h} P\right) \Delta w_{s} \beta\left(v_{h} P\right)\right)+\mu\left(\beta\left(v_{h} P\right) \Delta w_{h} \beta(P)\right) \\
& =\mu\left(\beta\left(v_{s h} P\right) \Delta w_{s} \beta\left(v_{h} P\right)\right)+\epsilon|h| /(2|F|) .
\end{aligned}
$$

Next we observe that

$$
\begin{aligned}
\mu & \left(\beta\left(v_{s h} P\right) \Delta w_{s} \beta\left(v_{h} P\right)\right) \\
& =\sum_{P_{1}, P_{2}} \mu\left(\beta\left(P_{1}\right) \cap w_{s} \beta\left(P_{2}\right)\right)+\sum_{P_{3}, P_{4}} \beta\left(P_{3} \cap w_{s} \beta\left(P_{4}\right)\right)
\end{aligned}
$$

where the first sum is over all $P_{1}, P_{2} \in \mathcal{P}^{\prime}$ such that $P_{1} \subset v_{s h} P$ and $P_{2} \cap v_{h} P=\emptyset$ while the second sum is over all $P_{3}, P_{4} \in \mathcal{P}^{\prime}$ such that $P_{3} \cap v_{s h} P=\emptyset$ and $P_{4} \subset v_{h} P$. 
By $(5)$ if $(i, j)=(1,2)$ or $(i, j)=(3,4)$ as above then

$$
\mu\left(\beta\left(P_{i}\right) \cap w_{s} \beta\left(P_{j}\right)\right)<\mu\left(P_{i} \cap v_{s} P_{j}\right)+\epsilon\left|\mathcal{P}^{\prime}\right|^{-2} /(4|F|)=\epsilon\left|\mathcal{P}^{\prime}\right|^{-2} /(4|F|) .
$$

Therefore,

$$
\mu\left(\beta\left(v_{s h} P\right) \Delta w_{s} \beta\left(v_{h} P\right)\right)<\epsilon /(2|F|) .
$$

This implies the claim.

Next we verify (4) with $Q_{i}=\beta\left(P_{i}\right)$ :

$$
\begin{aligned}
\mid \mu & \left(P_{i} \cap v_{g} P_{j}\right)-\mu\left(Q_{i} \cap w_{g} Q_{j}\right) \mid \\
& =\left|\mu\left(P_{i} \cap v_{g} P_{j}\right)-\mu\left(\beta\left(P_{i}\right) \cap w_{g} \beta\left(P_{j}\right)\right)\right| \\
& <\left|\mu\left(P_{i} \cap v_{g} P_{j}\right)-\mu\left(\beta\left(P_{i}\right) \cap \beta\left(v_{g} P_{j}\right)\right)\right|+\epsilon|g| /(2|F|) \\
& =\left|\mu\left(P_{i} \cap v_{g} P_{j}\right)-\mu\left(\beta\left(P_{i} \cap v_{g} P_{j}\right)\right)\right|+\epsilon|g| /(2|F|) \\
& <\epsilon / 2+\epsilon|g| /(2|F|) \\
& \leq \epsilon .
\end{aligned}
$$

The first inequality follows from Claim 1 and the second inequality from Claim 2 .

\section{A combinatorial lemma}

The next lemma will be used to rearrange partial orbits of a single transformation. Roughly speaking it states that any partial orbit which is roughly equidistributed with respect to some partition can be rearranged so as to approximate the local statistics of any given Markov chain on the partition.

Lemma 3.1. Let $\mathcal{A}$ be a finite set, $\pi$ be a probability distribution on $\mathcal{A}$ and $\mathcal{J}$ be a self-coupling of $\pi$ (so $\mathcal{J}$ is a probability distribution on $\mathcal{A} \times \mathcal{A}$ such that the projection of $\mathcal{J}$ to either factor is $\pi$ ). Let $0<\epsilon<1$ and $N>0$ be an integer and $\phi:\{1, \ldots, N\} \rightarrow \mathcal{A}$ a map such that if $\pi^{\prime}$ is the empirical distribution of $\phi$ then $\left\|\pi^{\prime}-\pi\right\|_{\infty}<\epsilon$. (By definition, $\pi^{\prime}=\phi_{*} u_{N}$ where $u_{N}$ is the uniform probability distribution on $\{1, \ldots, N\})$. We assume $\min _{a, b \in \mathcal{A}} \mathcal{\partial}(a, b)>2|\mathcal{A}| \epsilon+|\mathcal{A}|^{2} / N$.

Then there exists a bijection

$$
\sigma=\sigma_{\phi}:\{1, \ldots, N-1\} \longrightarrow\{2, \ldots, N\}
$$

such that if $\Gamma(\sigma)$ is the graph with vertices $\{1, \ldots, N\}$ and edges $\{(i, \sigma(i))$ : $1 \leq i \leq N-1\}$ then 
- $\Gamma(\sigma)$ is connected (so it is isomorphic to a line graph)

- if $\Phi_{\sigma}:\{1, \ldots, N-1\} \rightarrow \mathcal{A} \times \mathcal{A}$ is the map $\Phi_{\sigma}(i)=(\phi(i), \phi(\sigma(i)))$ and $\mathcal{J}_{\sigma}=\left(\Phi_{\sigma}\right)_{*} u_{N-1}$ is the empirical distribution of $\Phi_{\sigma}$ then

$$
\left\|\partial_{\sigma}-\mathcal{\partial}\right\|_{\infty}<2|\mathcal{A}| \epsilon+3|\mathcal{A}|^{2} / N
$$

To prove Lemma 3.1 we need some preliminary results.

Claim 1. There exists a self-coupling $\mathrm{g}^{\prime}$ of $\pi^{\prime}$ such that

$$
\left\|\mathcal{J}^{\prime}-\mathcal{\partial}\right\|_{\infty}<2|\mathcal{A}| \epsilon+|\mathcal{A}|^{2} / N
$$

and $\mathrm{J}^{\prime}$ takes values only in $\mathbb{Z}[1 / N]$.

Proof of Claim 1. Let $a \in \mathcal{A}$. For $b, c \in \mathcal{A} \backslash\{a\}$, let $\mathcal{J}^{\prime}(b, c)$ be the closest number in $\mathbb{Z}[1 / N]$ to $\mathcal{J}(b, c)$. Define

$$
\begin{aligned}
& J^{\prime}(a, c)=\pi^{\prime}(c)-\sum_{t \in \mathcal{A} \backslash\{a\}} J^{\prime}(t, c), \\
& J^{\prime}(b, a)=\pi^{\prime}(b)-\sum_{t \in \mathcal{A} \backslash\{a\}} J^{\prime}(b, t), \\
& \mathcal{J}^{\prime}(a, a)=\pi^{\prime}(a)-\sum_{t \in \mathcal{A} \backslash\{a\}} J^{\prime}(a, t)=\pi^{\prime}(a)-\sum_{t \in \mathcal{A} \backslash\{a\}} J^{\prime}(t, a) .
\end{aligned}
$$

It is straightforward to check that

$$
\left\|\mathcal{J}^{\prime}-\mathcal{J}\right\|_{\infty}<\epsilon+|\mathcal{A}|(\epsilon+|\mathcal{A}| / N) \leq 2|\mathcal{A}| \epsilon+|\mathcal{A}|^{2} / N
$$

Because $\min _{a, b \in \mathcal{A}} \mathcal{J}(a, b)>2|\mathcal{A}| \epsilon+|\mathcal{A}|^{2} \epsilon / N$, this implies $\mathcal{J}^{\prime}$ is positive everywhere. So it is a self-coupling of $\pi^{\prime}$.

Claim 2. There exists a bijection

$$
\tau:\{1, \ldots, N-1\} \longrightarrow\{2, \ldots, N\}
$$

such that if $\Phi_{\tau}:\{1, \ldots, N\} \rightarrow \mathcal{A} \times \mathcal{A}$ is the map $\Phi_{\tau}(i)=(\phi(i), \phi(\tau(i)))$ and $\mathcal{J}_{\tau}=\left(\Phi_{\tau}\right)_{*} u_{N}$ is the empirical distribution of $\Phi_{\tau}$ then

$$
\left\|\mathcal{\partial}_{\tau}-\mathcal{J}^{\prime}\right\|_{\infty} \leq 1 / N
$$


Proof of Claim 2. Because $\mathcal{J}^{\prime}$ is a self-coupling of $\pi^{\prime}$ taking values in $\mathbb{Z}[1 / N]$ there exist partitions $\mathcal{P}=\left\{P_{a, b}\right\}_{a, b \in \mathcal{A}}, \mathcal{Q}=\left\{Q_{a, b}\right\}_{a, b \in \mathcal{A}}$ of $\{1, \ldots, N\}$ such that

- $\left|P_{a, b}\right|=\left|Q_{a, b}\right|=N \mathcal{J}^{\prime}(a, b)$ for every $a, b \in \mathcal{A}$;

- $P_{a, b} \subset \phi^{-1}(a)$ and $Q_{a, b} \subset \phi^{-1}(b)$ for every $a, b \in \mathcal{A}$.

Next we choose bijections $\beta_{a, b}: P_{a, b} \rightarrow Q_{a, b}$ for all $a, b \in \mathcal{A}$. Define

$$
\tau:\{1, \ldots, N-1\} \longrightarrow\{2, \ldots, N\}
$$

by

$$
\tau(i)= \begin{cases}\beta_{a, b}(i) & \text { if } i \in P_{a, b} \text { and } \beta_{a, b}(i) \neq 1, \\ N & \text { if } i \in P_{a, b}(\text { for some } a, b) \text { and } \beta_{a, b}(i)=1 .\end{cases}
$$

This satisfies the claim.

Let $\tau:\{1, \ldots, N-1\} \rightarrow\{2, \ldots, N\}$ be a bijection satisfying the conclusion of Claim 2 with the property that the number of connected components of the graph $\Gamma(\tau)$ is as small as possible given that $\tau$ satisfies Claim 2 .

Claim 3. $\Gamma(\tau)$ has at most $|\mathcal{A}|^{2}$ connected components.

Proof of Claim 3. To obtain a contradiction, suppose $\Gamma(\tau)$ has more than $|\mathcal{A}|^{2}$ connected components. Then there exists $1 \leq i<j \leq N-1$ such that $i$ and $j$ are in different connected components of $\Gamma(\tau), \phi(i)=\phi(j)$ and $\phi(\tau(i))=\phi(\tau(j))$. Let us define

$$
\tau^{\prime}:\{1, \ldots, N-1\} \longrightarrow\{2, \ldots, N\}
$$

by

$$
\tau^{\prime}(k)= \begin{cases}\tau(k) & \text { if } k \notin\{i, j\}, \\ \tau(j) & \text { if } k=i, \\ \tau(i) & \text { if } k=j .\end{cases}
$$

Observe that $\tau^{\prime}$ also satisfies Claim 2 and $\Gamma\left(\tau^{\prime}\right)$ has one fewer connected component than $\Gamma(\tau)$ contradicting the choice of $\Gamma(\tau)$.

Proof of Lemma 3.1. Let $1 \leq i_{1}<i_{2}<\ldots<i_{k} \leq N$ be a maximal set of indices such that for $t \neq s, i_{t}$ and $i_{s}$ are in different connected components of $\Gamma(\tau)$. Define the bijection

$$
\sigma:\{1, \ldots, N-1\} \longrightarrow\{2, \ldots, N\}
$$


by

$$
\sigma(t)= \begin{cases}\tau(t) & \text { if } t \notin\left\{i_{1}, \ldots, i_{k}\right\}, \\ \tau\left(i_{s+1}\right) & \text { if } \left.t=i_{s} \text { (indices } \bmod k\right) .\end{cases}
$$

Observe that $\Gamma(\sigma)$ is connected and, since $k \leq|\mathcal{A}|^{2}$,

$$
\begin{aligned}
\left|\partial_{\sigma}(a, b)-\partial_{\tau}(a, b)\right| & =\left|\frac{\#\{1 \leq i \leq N-1: \phi(i)=a, \phi(\sigma(i))=b\}}{N}-\partial_{\tau}(a, b)\right| \\
& \leq|\mathcal{A}|^{2} / N .
\end{aligned}
$$

This implies the lemma.

\section{Proof of Theorem 1.1}

From here on, it will be convenient to work with observables instead of partitions. So instead of considering partitions $\mathcal{P}$ of a space $X$ we consider measurable maps $\phi: X \rightarrow \mathcal{A}$ where $\mathcal{A}$ is a finite set. Of course, $\left\{\phi^{-1}(\{a\}): a \in \mathcal{A}\right\}$ is the partition of $X$ represented by $\phi$.

Lemma 4.1. Let $(X, \mu)$ be a standard probability space and $T \in \operatorname{Aut}(X, \mu)$ be aperiodic. Let $\psi: X \rightarrow \mathcal{A}$ be a measurable map into a finite set. Let $\mu=$ $\int \nu d \omega(v)$ be the ergodic decomposition of $\mu$ with respect to $T$ (so $\omega$ is a probability measure on the space of $T$-invariant ergodic Borel probability measures on $X$ ). Suppose that for some $1 / 6>\epsilon>0$,

$$
\omega\left(\left\{v:\left\|\psi_{*} v-\psi_{*} \mu\right\|_{\infty}>\epsilon\right\}\right)<\epsilon .
$$

Suppose also that $\mathcal{J}$ is a self-coupling of $\psi_{*} \mu$ (i.e. $\mathcal{J}$ is a probability measure on $\mathcal{A} \times \mathcal{A}$ whose projections are both equal to $\psi_{*} \mu$ ) and

$$
\min _{a, b \in \mathcal{A}} \mathcal{J}(a, b)>2|\mathcal{A}| \epsilon .
$$

Then there exists $T^{\prime} \in \operatorname{Aut}(X, \mu)$ such that $T$ and $T^{\prime}$ have the same orbits (a.e.) and if $\Phi: X \rightarrow \mathcal{A} \times \mathcal{A}$ is the map $\Phi(x)=\left(\psi(x), \psi\left(T^{\prime} x\right)\right)$ then

$$
\left\|\Phi_{*} \mu-\mathcal{J}\right\|_{\infty} \leq 9|\mathcal{A}| \epsilon .
$$


Proof. By the pointwise ergodic theorem, there exists a Borel set $X^{\prime} \subset X$ and an integer $M>0$ such that

- $\mu\left(X^{\prime}\right)>1-\epsilon$,

- for every $x \in X^{\prime}$, every $a \in \mathcal{A}$ and every $K_{1}, K_{2} \geq M$,

$$
\left|\frac{\#\left\{-K_{1} \leq j \leq K_{2}: \psi\left(T^{j} x\right)=a\right\}}{K_{1}+K_{2}+1}-\psi_{*} \mu(a)\right|<\epsilon .
$$

Without loss of generality, we may assume $M$ is large enough so that

$$
\min _{a, b \in \mathcal{A}} \mathcal{J}(a, b)>2|\mathcal{A}| \epsilon+|\mathcal{A}|^{2} /(2 M+1)
$$

and $3|\mathcal{A}|^{2} /(2 M+1)<\epsilon$.

Let $Y \subset X$ be a complete section with $\mu(Y) \leq \epsilon /(2 M+1)$. By a complete section we mean that for $\mu$-a.e. $x \in X$ the orbit of $x$ intersects $Y$ nontrivially. The existence of such a complete section is proved in [20, Chapter II, Lemma 6.7]. Without loss of generality, we will assume that if $y \in Y$ then $T y \notin Y$.

For any integer $N \geq 1$ let us say that a map $\eta:\{1, \ldots, N\} \rightarrow \mathcal{A}$ is $\epsilon$-good if

$$
\left\|\eta_{*} u_{N}-\psi_{*} \mu\right\|_{\infty}<\epsilon
$$

and

$$
\min _{a, b \in \mathcal{A}} \mathcal{J}(a, b)>2|\mathcal{A}| \epsilon+|\mathcal{A}|^{2} / N
$$

where $u_{N}$ is the uniform probability measure on $\{1, \ldots, N\}$. For each $\epsilon$-good map

$$
\eta:\{1, \ldots, N\} \rightarrow \mathcal{A}
$$

choose a map

$$
\sigma_{\eta}:\{1, \ldots, N-1\} \longrightarrow\{2, \ldots, N\}
$$

as in Lemma 3.1.

For $x \in X$, let $\alpha(x)$ be the smallest nonnegative integer such that $T^{-\alpha(x)} x \in Y$, let $\beta(x)$ be the smallest nonnegative integer such that $T^{\beta(x)} x \in Y$ and let

$$
\psi_{x}:\{1, \ldots, \alpha(x)+\beta(x)+1\} \longrightarrow \mathcal{A}
$$

be the map

$$
\psi_{x}(j)=\psi\left(T^{j-\alpha(x)-1} x\right)
$$

So

$$
\psi_{x}=\left(\psi\left(T^{-\alpha(x)} x\right), \psi\left(T^{-\alpha(x)+1} x\right), \ldots, \psi\left(T^{\beta(x)} x\right)\right) .
$$


Note that $\psi_{x}$ is $\epsilon$-good if $x \in X^{\prime}, \alpha(x) \geq M$ and $\beta(x) \geq M$. In this case, let $\sigma_{x}=\sigma_{\psi_{x}}$ and $\mathcal{J}_{x}=\mathcal{J}_{\sigma_{x}}$ (with notation as in Lemma 3.1).

Now we can define $T^{\prime}$ as follows:

$$
T^{\prime} x= \begin{cases}T x & \text { if either } x \in Y \text { or } \psi_{x} \text { is not } \epsilon \text {-good } \\ T^{\sigma_{x}(\alpha(x)+1)-\alpha(x)-1}(x) & \text { otherwise. }\end{cases}
$$

Because each $\sigma_{x}$ is a bijection and the graph $\Gamma\left(\sigma_{x}\right)$ is connected it immediately follows that $T^{\prime}$ and $T$ have the same orbits.

By Kac's Theorem (see for example [6, Theorem 4.3.4]),

$$
\int_{Y} \beta(T x)+1 d \mu(x)=1 .
$$

Therefore the set $Y^{\prime}$ of all $x \in Y$ such that $\beta(T x)+1 \geq 2 M+1$ satisfies

$$
\begin{aligned}
\int_{Y^{\prime}} \beta(T x)+1 d \mu(x) & =1-\int_{Y \backslash Y^{\prime}} \beta(T x)+1 d \mu(x) \\
& \geq 1-\mu(Y)(2 M+1) \\
& \geq 1-\epsilon .
\end{aligned}
$$

Let $X^{\prime \prime}$ be the set of all $T^{j} x$ for $x \in Y^{\prime}$ and $M \leq j \leq \beta(T x)-M$. Then

$$
\begin{aligned}
\mu\left(X^{\prime \prime}\right) & =\int_{Y^{\prime}}(\beta(T x)-2 M+1) d \mu(x) \\
& \geq 1-\epsilon-\mu\left(Y^{\prime}\right)(2 M) \\
& \geq 1-2 \epsilon .
\end{aligned}
$$

Let

$$
X^{\prime \prime \prime}=X^{\prime} \cap X^{\prime \prime} .
$$

So $\mu\left(X^{\prime \prime \prime}\right) \geq 1-3 \epsilon$. Observe that if $x \in X^{\prime \prime \prime}$ then $\alpha(x) \geq M$ and $\beta(x) \geq M$ so $\psi_{x}$ is $\epsilon$-good. Finally, let $Y^{\prime \prime}$ be the set of all $y \in Y^{\prime}$ such that $T^{j} y \in X^{\prime \prime \prime}$ for some $1 \leq j \leq \beta(T x)+1$. Then

$$
\int_{Y^{\prime \prime}} \beta(T x)+1 d \mu(x) \geq \mu\left(X^{\prime \prime \prime}\right) \geq 1-3 \epsilon .
$$

If $y \in Y^{\prime \prime}$, then $\psi_{T y}$ is $\epsilon$-good (this uses our hypothesis that if $y \in Y^{\prime \prime} \subset Y$ then $T y \notin Y)$. Let $Z$ be the set of all $T^{j} y$ for $y \in Y^{\prime \prime}$ and $0 \leq j \leq \beta(T y)$. So

$$
\mu(Z)=\int_{Y^{\prime \prime}} \beta(T x)+1 d \mu(x) \geq 1-3 \epsilon .
$$


Recall that $\Phi: X \rightarrow \mathcal{A} \times \mathcal{A}$ is defined by $\Phi(x)=\left(\psi(x), \psi\left(T^{\prime} x\right)\right)$. Let $\mu_{Z}$ denote the unnormalized restriction of $\mu$ to $Z$. Then

$$
\begin{aligned}
\left\|\Phi_{*} \mu-\mathcal{\partial}\right\|_{\infty} & \leq\left\|\Phi_{*} \mu-\Phi_{*} \mu_{Z}\right\|_{\infty}+\left\|\Phi_{*} \mu_{Z}-\mathcal{\partial}\right\|_{\infty} \\
& \leq 3 \epsilon+\left\|\Phi_{*} \mu_{Z}-\mathcal{\partial}\right\|_{\infty} .
\end{aligned}
$$

Next observe that

$$
\Phi_{*} \mu_{Z}=\int_{Y^{\prime \prime}}[\beta(T x)+1] \mathcal{J}_{T x} d \mu(x) .
$$

By Lemma 3.1, for $x \in Y^{\prime \prime}$

$$
\left\|\mathcal{J}_{T x}-\mathcal{J}\right\|_{\infty} \leq 2|\mathcal{A}| \epsilon+3|\mathcal{A}|^{2} /(2 M+1) \leq 3|\mathcal{A}| \epsilon .
$$

So

$$
\left\|\Phi_{*} \mu_{Z}-\mathcal{J}\right\|_{\infty} \leq(3|\mathcal{A}| \epsilon) /(1-3 \epsilon) \leq 6|\mathcal{A}| \epsilon
$$

(because $\epsilon<1 / 6)$ and therefore,

$$
\left\|\Phi_{*} \mu-\mathcal{J}\right\|_{\infty} \leq 3 \epsilon+6|\mathcal{A}| \epsilon \leq 9|\mathcal{A}| \epsilon .
$$

In the next lemma, we prove the existence of a "good observable" $\psi$.

Lemma 4.2. Let $G$ be a countable group and $S \subset G$ a finite set of elements of infinite order. Let $T \in A(G, X, \mu)$ be an essentially free action of $G$. For each $s \in S$ let $\mu=\int \nu d \omega_{s}(v)$ be the ergodic decomposition of $\mu$ with respect to $T_{s}$ (so $\omega_{s}$ is a Borel probability measure on the space of all $T_{s}$-invariant ergodic Borel probability measures).

Let $\pi$ be a probability measure on a finite set $\mathcal{A}$. Also let $0<\epsilon<1 / 2$. Then there exists a measurable map $\psi: X \rightarrow \mathcal{A}$ such that for every $s \in S$,

$$
\omega_{s}\left(\left\{v:\left\|\psi_{*} v-\pi\right\|_{\infty}>3 \epsilon\right\}\right)<\epsilon \text {. }
$$

Proof. Given a measurable map $\psi: X \rightarrow \mathcal{A}$, an element $s \in S$ and an integer $N \geq 1$ let $\psi_{s, N}: X \rightarrow \mathcal{A}^{N}$ be the map

$$
\psi_{s, N}(x)=\left(\psi\left(T_{s} x\right), \psi\left(T_{s}^{2} x\right), \ldots, \psi\left(T_{s}^{N} x\right)\right) .
$$

According to Abert-Weiss [2], the action $T$ weakly contains the Bernoulli shift action $G \curvearrowright(\mathcal{A}, \pi)^{G}$. This immediately implies that for any integer $N \geq 1$ there exists a measurable map $\psi: X \rightarrow \mathcal{A}$ such that for every $s \in S$

$$
\left\|\left(\psi_{s, N}\right)_{*} \mu-\pi^{N}\right\|_{\infty}<\frac{\epsilon^{2}}{2} .
$$


Given a sequence $y \in \mathcal{A}^{N}$, let $E[y]$ denote its empirical distribution. To be precise, $E[y]$ is the probability measure on $\mathcal{A}$ defined by

$$
E[y](a)=\#\{1 \leq i \leq N: y(i)=a\} / N .
$$

By the law of large numbers we may choose $N$ large enough so that

$$
\pi^{N}\left(\left\{y \in \mathcal{A}^{N}:\|E[y]-\pi\|_{\infty}>\epsilon\right\}\right)<\epsilon^{2} / 2 .
$$

Therefore,

$$
\begin{aligned}
\mu\left(\left\{x \in X:\left\|E\left[\psi_{s, N}(x)\right]-\pi\right\|_{\infty}>\epsilon\right\}\right) \\
\quad=\left(\psi_{s, N}\right)_{*} \mu\left(\left\{y \in \mathcal{A}^{N}:\|E[y]-\pi\|_{\infty}>\epsilon\right\}\right) \\
\quad<\epsilon^{2}
\end{aligned}
$$

for every $s \in S$. Let

$$
Z=\left\{x \in X:\left\|E\left[\psi_{s, N}(x)\right]-\pi\right\|_{\infty}>\epsilon\right\} .
$$

So

$$
\int v(Z) d \omega_{s}(v)=\mu(Z)<\epsilon^{2}
$$

This implies $\omega_{s}(\{v: v(Z)>\epsilon\})<\epsilon$.

Next we claim that if a probability measure $v$ satisfies $v(Z) \leq \epsilon$ then

$$
\left\|\psi_{*} \nu-\pi\right\|_{\infty} \leq 3 \epsilon
$$

Indeed,

$$
\psi_{*} \nu=E_{*}\left(\psi_{s, N}\right)_{*} \nu
$$

So if $v(Z) \leq \epsilon$ then

$$
\left\|\psi_{*} v-E_{*}\left(\psi_{s, N}\right)_{*}\left(v \uparrow Z^{c}\right)\right\|_{\infty} \leq \epsilon
$$

(where $Z^{c}=X \backslash Z$ is the complement of $Z$ ) and

$$
\left\|\pi-E_{*}\left(\psi_{s, N}\right)_{*}\left(v \uparrow Z^{c}\right)\right\|_{\infty} \leq \frac{\epsilon}{1-\epsilon}
$$

by definition of $Z$. So $\left\|\psi_{*} \nu-\pi\right\|_{\infty} \leq 3 \epsilon$ (since we assume $\epsilon<1 / 2$ ).

Since $\omega_{s}(\{v: v(Z)>\epsilon\})<\epsilon$, we now have

$$
\omega_{s}\left(\left\{v:\left\|\psi_{*} v-\pi\right\|_{\infty}>3 \epsilon\right\}\right)<\epsilon .
$$


Proof of Theorem 1.1. Let $G$ be a finitely generated free group with free generating set $S \subset G$. Let $a, b \in A(G, X, \mu)$ and assume $a$ is essentially free. It suffices to show that $b \in \overline{[a]_{O E}}$. By Lemma 2.3 it suffices to show that for every finite set $\mathcal{A}$, measurable map $\phi: X \rightarrow \mathcal{A}$ and $\epsilon>0$ there exists a measurable map $\psi: X \rightarrow \mathcal{A}$ and $a^{\prime} \in[a]_{O E}$ such that

$$
\left\|\left(\psi \vee \psi \circ a_{s}^{\prime}\right)_{*} \mu-\left(\phi \vee \phi \circ b_{s}\right)_{*} \mu\right\|_{\infty} \leq 10|\mathcal{A}| \epsilon \quad \forall s \in S
$$

where, for example, $\phi \vee \phi \circ b_{s}: X \rightarrow \mathcal{A} \times \mathcal{A}$ is the map

$$
\phi \vee \phi \circ b_{s}(x)=\left(\phi(x), \phi\left(b_{s} x\right)\right) .
$$

After replacing $\mathcal{A}$ with the essential range of $\phi$ if necessary, we may assume that $\phi_{*} \mu(c)>0$ for every $c \in \mathcal{A}$. We claim that there exists a self-coupling $\mathcal{J}_{s}$ of $\phi_{*} \mu$ such that $\mathcal{J}_{s}(c, d)>0$ for all $c, d \in \mathcal{A}$ and

$$
\left\|\left(\phi \vee \phi \circ b_{s}\right)_{*} \mu-\mathcal{J}_{s}\right\|_{\infty}<\epsilon .
$$

Indeed, the self-coupling

$$
\partial_{s}=(1-\epsilon)\left(\phi \vee \phi \circ b_{s}\right)_{*} \mu+\epsilon(\phi \times \phi)_{*}(\mu \times \mu)
$$

has this property. After choosing $\epsilon$ smaller if necessary we may assume that $\epsilon<1 / 6$ and

$$
\min _{s \in S} \min _{c, d \in \mathcal{A}} \partial_{s}(c, d)>2|\mathcal{A}| \epsilon .
$$

Let $\mu=\int \nu d \omega_{s}$ be the ergodic decomposition of $\mu$ with respect to $a_{s}$. By Lemma 4.2 there exists a measurable map $\psi: X \rightarrow \mathcal{A}$ such that

$$
\omega_{s}\left(\left\{v:\left\|\phi_{*} \mu-\psi_{*} v\right\|_{\infty}>\epsilon\right\}\right)<\epsilon
$$

for every $s \in S$. By Lemma 4.1 for every $s \in S$ there exists $a_{s}^{\prime} \in \operatorname{Aut}(X, \mu)$ such that $a_{s}^{\prime}$ and $a_{s}$ have the same orbits and

$$
\left\|\left(\psi \vee \psi \circ a_{s}^{\prime}\right)_{*} \mu-\mathcal{J}_{s}\right\|_{\infty} \leq 9|\mathcal{A}| \epsilon .
$$

Because $a_{s}^{\prime}$ and $a_{s}$ have the same orbits for every $s \in S$ it follows that the homomorphism $a^{\prime}: G \rightarrow \operatorname{Aut}(X, \mu)$ defined by $\left\{a_{s}^{\prime}\right\}_{s \in S}$ is orbit-equivalent to $a$. In other words, $a^{\prime} \in[a]_{O E}$. Also

$$
\begin{aligned}
& \left\|\left(\psi \vee \psi \circ a_{s}^{\prime}\right)_{*} \mu-\left(\phi \vee \phi \circ b_{s}\right)_{*} \mu\right\|_{\infty} \\
& \quad \leq\left\|\left(\psi \vee \psi \circ a_{s}^{\prime}\right)_{*} \mu-\mathcal{J}_{s}\right\|_{\infty}+\left\|\mathcal{J}_{s}-\left(\phi \vee \phi \circ b_{s}\right)_{*} \mu\right\|_{\infty} \\
& \quad \leq 10|\mathcal{A}| \epsilon .
\end{aligned}
$$

By Lemma 1.6, $a^{\prime} \in A_{\text {free }}(G, X, \mu)$. This finishes the special case in which $G$ is finitely generated. The general case follows from Corollary 2.2. 


\section{References}

[1] M. Abért and G. Elek, Dynamical properties of profinite actions. Ergodic Theory Dynam. Systems 32 (2012), no. 6, 1805-1835. Zbl 1297.37004 MR 2995875

[2] M. Abért and B. Weiss, Ergodic Theory Dynam. Systems 33 (2013), no. 2, 323-333. Zbl 1268.37006 MR 3035287

[3] I. Benjamini, R. Lyons, and O. Schramm, Unimodular random trees. Ergodic Theory Dynam. Systems 35 (2015), no. 2, 359-373. Zbl 06423374 MR 3316916

[4] L. Bowen, Periodicity and packings of the hyperbolic plane. Geom. Dedicata 102 (2003), 213-236. Zbl 1074.52007 MR 2026846

[5] C. Conley, A. Kechris, and R. Tucker-Drob, Ultraproducts of measure preserving actions and graph combinatorics. Ergodic Theory Dynam. Systems 33 (2013), no. 2, 334-374. Zbl 1268.05104 MR 3035288

[6] T. Downarowicz, Entropy in dynamical systems. New Mathematical Monographs, 18. Cambridge University Press, Cambridge, 2011. Zbl 1220.37001 MR 2809170

[7] H. A. Dye, On groups of measure preserving transformation. I. Amer. J. Math. 81 (1959), 119-159. MR 0131516

[8] H. A. Dye, On groups of measure preserving transformations. II. Amer. J. Math. 85 (1963), 551-576. MR 0158048

[9] G. Elek and G. Lippner, Sofic equivalence relations. J. Funct. Anal. 258 (2010), no. 5, 1692-1708. Zbl 05674486 MR 2566316

[10] I. Epstein, Orbit inequivalent actions of non-amenable groups. Preprint 2007. arXiv:0707.4215 [math.GR]

[11] M. Foreman and B. Weiss, An anti-classification theorem for ergodic measure preserving transformations. J. Eur. Math. Soc. (JEMS) 6 (2004), no. 3, 277-292. Zbl 1063.37004 MR 2060477

[12] D. Gaboriau, Coût des relations d'équivalence et des groupes. Invent. Math. 139 (2000), no. 1, 41-98. Zbl 0939.28012 MR 1728876

[13] D. Gaboriau and S. Popa, An uncountable family of nonorbit equivalent actions of $\mathbb{F}_{n}$. J. Amer. Math. Soc. 18 (2005), no. 3, 547-559. Zbl 1155.37302 MR 2138136

[14] E. Glasner, J.-P. Thouvenot, and B. Weiss, Every countable group has the weak Rohlin property. Bull. London Math. Soc. 38 (2006), no. 6, 932-936. Zbl 1116.28013 MR 2285247

[15] G. Hjorth, A converse to Dye's theorem. Trans. Amer. Math. Soc. 357 (2005), no. 8, 3083-3103. Zbl 1068.03035 MR 2135736

[16] A. Ioana, A. S. Kechris, and T. Tsankov, Subequivalence relations and positivedefinite functions. Groups Geom. Dyn. 3 (2009), no. 4, 579-625. Zbl 1186.37011 MR 2529949

[17] A. Ioana, Non-orbit equivalent actions of $\mathbb{F}_{n}$. Ann. Sci. Éc. Norm. Supér. (4) 42 (2009), no. 4, 675-696. Zbl 1185.37009 MR 2568879 
[18] A. Kechris, Global aspects of ergodic group actions. Mathematical Surveys and Monographs, 160. American Mathematical Society, Providence, RI, 2010. Zbl 1189.37001 MR 2583950

[19] A. Kechris, Weak containment in the space of actions of a free group. Israel J. Math. 189 (2012), 461-507. Zbl 1273.37003 MR 2931406

[20] A. S. Kechris and B. D. Miller, Topics in orbit equivalence. Lecture Notes in Mathematics, 1852. Springer, Berlin, 2004. Zbl 1058.37003 MR 2095154

[21] A. Kechris and T. Tsankov, Amenable actions and almost invariant sets. Proc. Amer. Math. Soc. 136 (2008), no. 2, 687-697. Zbl 1155.28010 MR 2358510

[22] Y. Kida, Orbit equivalence rigidity for ergodic actions of the mapping class group. Geom. Dedicata 131 (2008), 99-109. Zbl 1194.37012 MR 2369194

[23] N. Monod and Y. Shalom, Orbit equivalence rigidity and bounded cohomology. Ann. of Math. (2) 164 (2006), no. 3, 825-878. Zbl 1129.37003 MR 2259246

[24] D. S. Ornstein and B. Weiss, Ergodic theory of amenable group actions. I. The Rohlin lemma. Bull. Amer. Math. Soc. (N.S.) 2 (1980), no. 1, 161-164. Zbl 0427.28018 MR 0551753

[25] S. Popa, Strong rigidity of $\mathrm{II}_{1}$ factors arising from malleable actions of $w$-rigid groups. II. Invent. Math. 165 (2006), no. 2, 409-451. Zb1 1120.46044 MR 2231962

[26] S. Popa, On the superrigidity of malleable actions with spectral gap. J. Amer. Math. Soc. 21 (2008), no. 4, 981-1000. Zbl 1222.46048 MR 2425177

[27] K. Schmidt, Amenability, Kazhdan's property T, strong ergodicity and invariant means for ergodic group actions. Ergodic Theory Dynamical Systems 1 (1981), no. 2, 223-236. Zbl 0485.28019 MR 0661821

Received August 29, 2013

Lewis Bowen, Mathematics Department, 1 University Station C1200, University of Texas at Austin, Austin, TX 78712, USA

e-mail: lpbowen@math.utexas.edu 